\title{
Thermo-mechanical load interactions in foam cored axi-symmetric sandwich circular plates - High-order and FE models
}

\author{
Carlos Santiuste $^{\mathrm{a}, *}$, Ole T. Thomsen ${ }^{\mathrm{b}}$, Yeoshua Frostig ${ }^{\mathrm{c}}$ \\ a Department of Continuum Mechanics and Structural Analysis, University Carlos III of Madrid, 28911 Leganés, Spain \\ ${ }^{\mathrm{b}}$ Department of Mechanical and Manufacturing Engineering, Aalborg University, DK-9220 Aalborg, Denmark \\ c Faculty of Civil and Environmental Engineering, Department of Structural Engineering, Technion - Israel Institute of Technology, Haifa 32000, Israel
}

Keywords:

Sandwich structures

Temperature-dependent mechanical

properties

Properties degradation

Flexible core

FEA modeling

High-order sandwich panel theory (HSAPT)

model

\begin{abstract}
A B S T R A C T
This work presents analytical and finite element analysis (FEA) results of the thermo mechanical non linear response of an axi symmetric circular sandwich plates with a compliant foam core. The study investigates the load thermal interaction response of a sandwich panel where the properties of the core are temperature dependent and degrade as the temperatures are raised. It presents briefly the governing equations for a sandwich plate based on the principles of the high order sandwich panel theory (HSAPT) which incorporates the effects of the vertical flexibility of the core material as well as the effects of tem perature independent/dependent mechanical properties of the foam core. The effects of the thermal deg radation of core material on the thermo mechanical non linear response of a simply supported circular sandwich plate are studied through the analytical and FE models. The difficulties involved in non linear geometrical FE modeling of sandwich panels with a compliant "soft" core with temperature dependent mechanical properties are discussed. The HSAPT model predictions are compared very well with FE result. An important conclusion of the study is that the interaction between mechanical loads, tempera ture induced deformations, and degradation of the mechanical properties due to elevated temperatures, may seriously affect the structural integrity of foam cored sandwich plates.
\end{abstract}

\section{Introduction}

Polymer foam cored sandwich structures are being used increasingly for a variety of applications including wind turbine blades, boat hulls and ship structures as well as for structural applications in the transportation and aerospace sectors, Zenkert [1]. They are used as primary and secondary structural compo nents, and their popularity is a result of their superior performance in terms of strength and stiffness to weight ratios, ease of manufac turing, and also optional characteristics such as acoustic and thermal insulation, their repair capability and flexibility in design. Polymer foam cored sandwich structures are often subjected to aggressive service condition which may include warm or elevated temperatures. The mechanical properties of polymer foam cores may degrade significantly with warm/elevated temperatures which may cause significant changes in the mechanical properties within the operating range of temperatures. For example, PVC foams (Divinycell ${ }^{\circledR}[2]$ ) lose all their stiffness and strength at about $80100{ }^{\circ} \mathrm{C}$, while PMI foams (Rohacell ${ }^{\circledR}$ [3]) lose the resistance at

\footnotetext{
* Corresponding author. Tel.: +34 916249920; fax: +34 916249973.

E-mail addresses: csantius@ing.uc3m.es (C. Santiuste), ott@me.aau.dk (O.T. Thomsen), cvrfros@techunix.technion.ac.il (Y. Frostig).
}

about $200{ }^{\circ} \mathrm{C}$. Moreover, the degradation of the mechanical proper ties may be associated with loss of stability at much lower temper atures than the temperatures which cause a complete loss of stiffness and strength.

The thermal degradation of polymer foam cored sandwich structures and its effects on the load thermal interaction response is generally poorly understood by researchers and industry. However, there is a growing concern within the wind turbine blade, marine and aeronautical sectors that the simultaneous action of mechanical loads and elevated temperatures may com promise the structural integrity under certain circumstances. At the same time the manufacturers of polymer foam core materials offer limited and incomplete information about the temperature dependency of the core properties as well the effects of interacting mechanical and thermal loads.

A large variety of analytical and numerical models are available for sandwich structures (beams, plates and shells). These models are usually based on the "equivalent single layer" approach (ESL approach), where the layered sandwich structure is represented by a solid panel with equivalent homogenized properties, Mindlin [4], Reddy [5]. Several researchers have used high order theories to model the thermo mechanical response of laminated monolithic or sandwich panels including Najafizadeh and Heydari [6], Dafedar 
and Desai [7], Kapuria and Achary [8], Shiau and Kuo [9], Matsunaga [10], Dawe [11] and Cooke [12]. However, none of these have in cluded the core through thickness flexibility. A more comprehen sive approach is the so called high order sandwich panel theory (HSAPT), which models the layered sandwich panel as composed of two face sheets and a core material that are interconnected through the equilibrium and compatibility conditions, and which incorporate the through thickness flexibility of the core.

The HSAPT approach has been successfully used for the analysis of sandwich beams and unidirectional panels and plates in several studies, including hygrothermal effects, buckling (global and local) and non linear response, see Frostig [13], Frostig and Thomsen [14], and Frostig et al. [15]. More recently, Frostig and Thomsen have studied thermal buckling and post buckling or non linear geometrically foam cored sandwich panels by adopting the HSAPT approach, and by assuming core properties that are either temper ature independent [16] or temperature dependent [17] and [18]. Temperature dependent face sheet properties can be included in the analyses, but as polymer foam cores are much more tempera ture sensitive than typical face sheet materials (composite lami nates or metallic faces) only temperature dependent core properties were included. Frostig and Thomsen [16 18] show that thermal degradation (softening) of polymer foam core materials exerts a significant influence on the performance of sandwich beams and unidirectional panels. More specifically, the degrada tion of the core properties with rising temperature lowers the buckling resistance, see Frostig and Thomsen [16,17]. In addition, when external mechanical loads act simultaneously with thermal loads, the material degradation may shift the response from being linear and stable into being non linear and unstable. This is espe cially pronounced when thermal gradients are present across the sandwich panel thickness, see Frostig and Thomsen [18].

The HSAPT models used in Frostig and Thomsen [16 18] en ables the non linear analysis of unidirectional sandwich structures of either beam (narrow) or panel (wide) type. However, practical engineering applications of sandwich structures nearly always in volve sandwich plate type structures, which are characterized by complex 3D stress and displacement/deformation states.

In this work, the HSAPT approach has been extended to analyze the behavior of axi symmetric circular plates including the core vertical flexibility and temperature dependent material properties.

In order to obtain a better understanding of the thermo mechanical non linear response of circular sandwich plates with a compliant foam core, the analyses developed in this work include a non linear HSAPT analytical model and non linear a finite ele ment analysis (FEA) model developed in the ABAQUS/Standard code. The combination of these two approaches provides the advantages of the analytical models, including the ability to de scribe the physical behavior of the sandwich structure explicitly, and the possibilities for conducting parametric studies and numer ical convergence even at very high face core stiffness ratios; but also the well known advantages of convergent FEA numerical mod els including the possibility to modify the plate geometry easily, the ability to model complex geometric shapes, complex loads and complex boundary conditions, the easy implementation of non linear material behavior, and finally the potential use in industrial applications for which the HSAPT approach must be con sidered intractable. On the other hand, the use of FEA in the design of foam cored sandwich structures presents certain difficulties including numerical instabilities due to the extremely large stiff ness ratios between the face sheets and the core materials (the ra tio of the Young's moduli of the face sheets and the core are typically in the range of $10^{2} 10^{3}$ at room temperature). In the interfaces between the core and the face sheets there are adjacent elements with large stiffness differences, and this will typically lead to significant element distortion which in addition leads to significant numerical difficulties in the FEA solution procedure. One of the difficulties that is revealed when modeling polymer foam cored sandwich plates with degrading mechanical properties is that the face core stiffness ratio increases with temperature as the polymer foam core loses its stiffness (softens) Hence, it may be anticipated that the convergence of the FEA solution may be come increasingly difficult as the temperature is increased. Thus, some of the well known advantages of the FEA method when com pared to analytical techniques may cease to exist as a result of the numerically unstable numerical solution that will occur when the face core stiffness ratio becomes very large. Under such circum stances the quality of the FEA solution is dubious, and a thorough convergence study has to be conducted. Some of the mentioned numerical problems are discussed in detail in this paper.

\section{Non-linear HSAPT equations for a circular sandwich plate}

The special case of an axi symmetric circular sandwich plate subjected to axi symmetric loads and boundary conditions is con sidered; see Fig. 1 for sign conventions and general notation. The governing equations are derived using the variational principle of extremum of the total potential energy, see Frostig and Thomsen [19] for details. In addition, kinematic relations corresponding to moderately displacements are adopted for the face sheets, whereas linear strain displacement is assumed for the core. The assump tions adopted here are those of a sandwich panel with a soft (ver tically) compressible core, see Frostig and Thomsen [18]. In addition, the formulation ahead assumes that the face sheets and the core are isotropic and linear elastic and only vertical distrib uted loads are considered. Hence, the governing equations for the axi symmetric circular sandwich plate case that include the equi librium equations for the face sheets (see Fig. 1), Eqs. (1) (6) ahead, the radial compatibility condition of full bond between the core and the lower face sheet, Eqs. (7) and (8), assuming here that the mechanical properties of the core material are temperature inde pendent, read $(j=t, b)$ :

$$
\begin{aligned}
& \frac{d}{d r} N_{r r_{j}}(r) \quad(1)^{k} \tau_{r}(r)+\frac{1}{2} \\
& \times \frac{\left(2 N_{r r_{j}}(r)+\alpha_{j} \mathbb{A}_{j}\left(T_{t b}(r)+T_{t t}(r)\right)\left(1+\mu_{j}\right)\right)\left(1+\mu_{j}\right)}{r} \\
& +\frac{\mathbb{A}\left(1 \quad \mu_{j}^{2}\right) u_{o j}(r)}{r^{2}}
\end{aligned}
$$

$$
\frac{d}{d r} u_{o j}(r) \quad \frac{1}{2}\left(1+\mu_{j}\right)\left(T_{t b}(r)+T_{t t}(r)\right) \alpha_{j}+\frac{N_{r r_{j}}(r)}{\mathbb{A}_{j}} \quad \frac{1}{2} D w_{j}(r)^{2} \quad \frac{\mu_{j} u_{o j}(r)}{r}
$$

$$
\begin{aligned}
\frac{d}{d r} V_{r z_{j}}(r) & \frac{V_{r z_{j}}(r)}{r}+(1)^{k} \sigma_{z z j}(r) \quad q_{j}(r) \\
\frac{d}{d r} M_{r r_{j}}(r) \quad & \frac{1}{2} d_{j} \tau_{r}(r)+V_{r z_{j}}(r) \quad N_{r r_{j}}(r) D w_{j}(r) \\
+ & \frac{\left(1+\mu_{j}\right) M_{r r_{j}}(r)}{r}+\frac{\left(1+\mu_{j}^{2}\right) D w_{j}(r) \mathbb{D}_{j}}{r^{2}} \\
& \frac{\left(1+\mu_{j}\right)\left(1+\mu_{j}\right)\left(T_{t b}(r) \quad T_{t t}(r)\right) \mathbb{D}_{j} \alpha_{j}}{d_{j} r}
\end{aligned}
$$

$\frac{d}{d r} w_{j}(r) \quad D w_{j}(r)$$$
\frac{d}{d r} D w_{j}(r) \quad \frac{M_{r r_{j}}(r)}{\mathbb{D}_{j}} \quad \frac{\mu_{j} D w_{j}(r)}{r}+\frac{\left(T_{t b}(r) \quad T_{t t}(r)\right)\left(1+\mu_{j}\right) \alpha_{j}}{d_{j}}
$$

$\frac{d}{d r} \tau_{r}(r) \quad D \tau_{r}(r) E_{z c}$ 


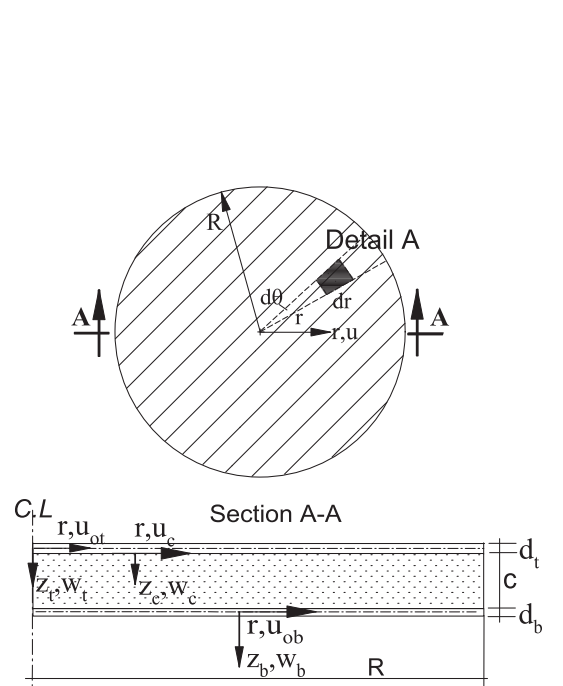

(a)

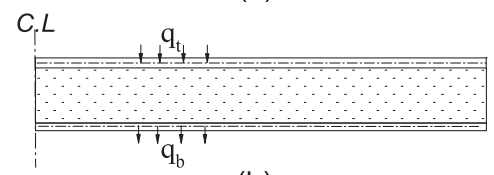

(b)
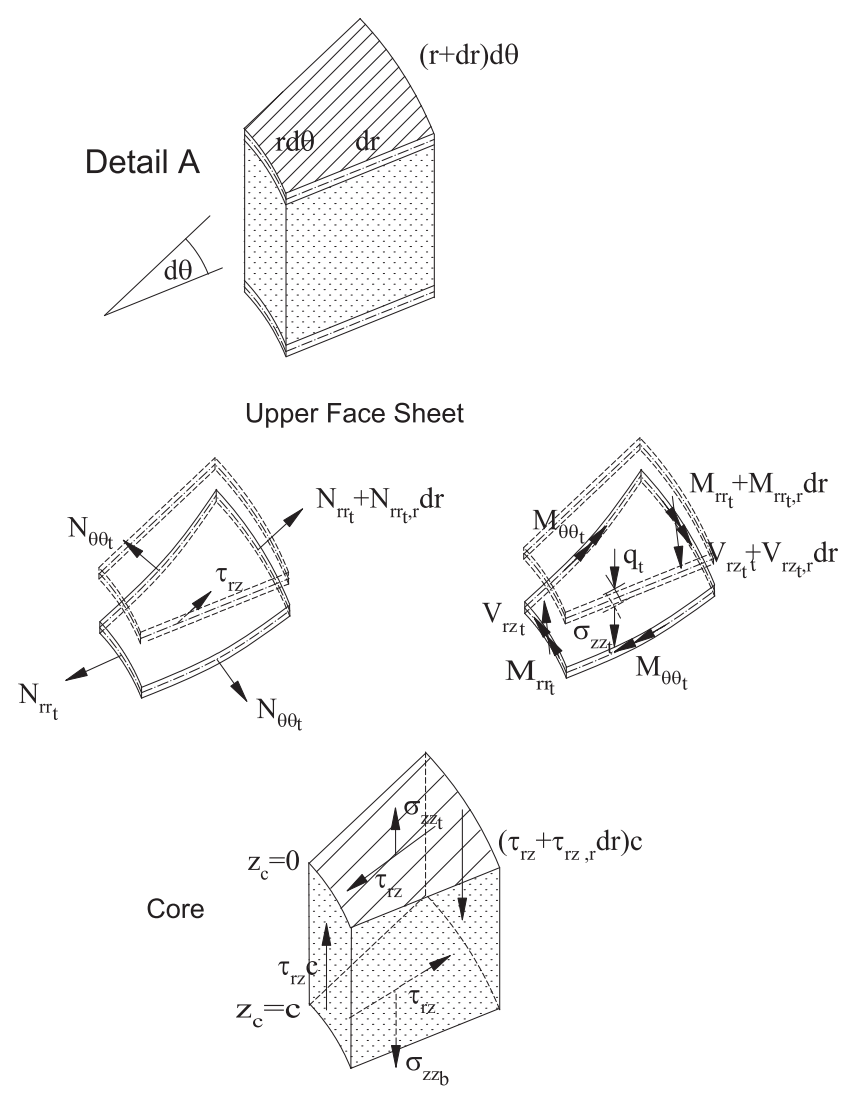

Lower Face Sheet
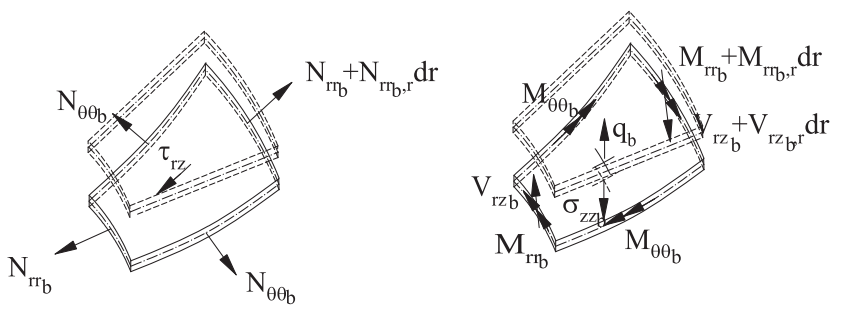

(c)

Fig. 1. A typical circular sandwich panel: (a) sign convention; (b) loads; (c) stress resultants in constituents.

$$
\begin{aligned}
& \frac{d}{d r} D \tau_{r}(r) \quad\left(\frac{12}{c^{2} G_{r z c}} \frac{1}{r^{2} E_{z c}}\right) \tau_{r}(r)+\left(\begin{array}{cc}
\frac{6 d_{b}}{c^{3}} & \frac{6}{c^{2}}
\end{array}\right) D w_{b}(r) \\
& +\left(\begin{array}{cc}
\frac{6}{c^{2}} & \frac{6 d_{t}}{c^{3}}
\end{array}\right) D w_{t}(r) \quad \frac{D \tau_{r}(r)}{r} \\
& +\frac{\left(\frac{d}{d r} T_{c b}(r)\left(\frac{d}{d r} T_{c t}(r)\right)\right) \alpha_{c}}{c}+\frac{12\left(u_{0} t(r) u_{o b}(r)\right)}{c^{3}}
\end{aligned}
$$

where $k=1$ for $j=t$ and 2 for $j=b ; N_{r r j}, V_{r z j}$ and $M_{r r j}(j=t, b)$ are the face sheet radial in plane and vertical shear stress resultants, and the bending moment stress resultants, respectively; $u_{o j}, w_{j}$, and $D w_{j}$ are the in plane and vertical displacements, and the radial rota tion of the centroid plane of the face sheets; respectively; $A_{j}, D_{j}$ and ${ }_{j}$ $(j=t, b)$ are the in plane and flexural rigidities, and the Poisson ratios, respectively, for the face sheets; $\alpha_{k}$ and $T_{k t}$ and $T_{k b}$ $(k=t, b, c)$ are the coefficients of thermal expansion, and the tem perature at the upper and the lower fiber of the faces and the core, respectively; $q_{j}(j=t, b)$ is the vertical distributed load, partially or fully distributed at the two face sheets; and $\tau_{r}$ and $D \tau_{r}$ are the radial shear stresses of the core and its gradient (slope) in the radial direc tion, respectively. Finally; $E_{z c}$ and $G_{r z c}$ are the vertical modulus of elasticity and shear modulus of the core in the $r$ direction. For sign conventions see Fig. 1. Please notice that the number of equations for the axi symmetric circular plate is 14 , similar to that of a unidirectional panel, see Frostig and Thomsen [18]. In addition, the axi symmetric constraints require that the shear stress in the circumferential direction to be null. And it yields identical in plane circumferential displacements at the centroid plane for the face sheets. For details see Frostig and Thomsen [19].

The circumferential stress resultants are determined through some algebraic manipulation and for the various face sheets they equal $(j=t, b)$ :

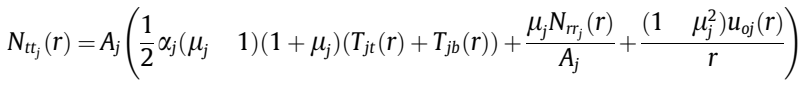

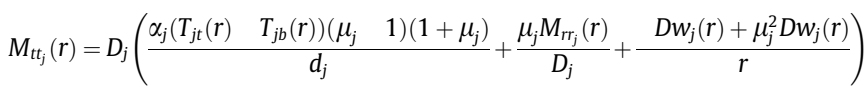

In addition, the vertical normal stresses at the upper and the lower face core interfaces that appear in the governing equations read: 


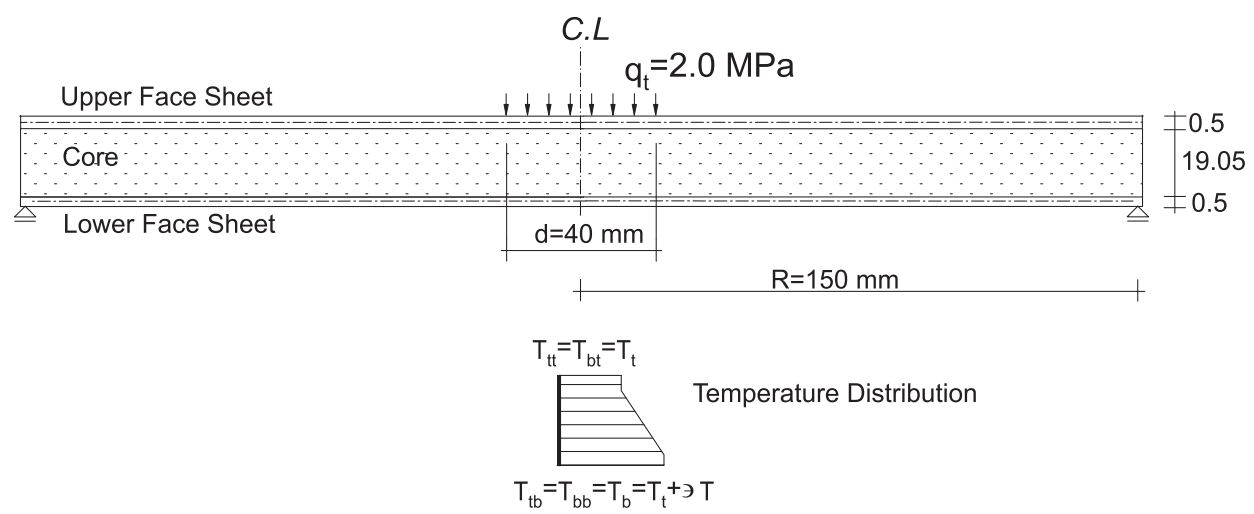

Fig. 2. Geometry and temperature distribution.

$$
\begin{aligned}
& \sigma_{z z b}(r) \quad \frac{1}{2} \frac{1}{c r}\left(c ^ { 2 } \tau _ { r } ( r ) \quad E _ { z c } r \left(\alpha_{c} c T_{c b}(r)+\alpha_{c} c T_{c t}(r) \quad 2 w_{b}(r)\right.\right. \\
& \left.\left.+2 w_{t}(r)+c^{2} D \tau_{r}(r)\right)\right), \sigma_{z z t}(r) \\
& \frac{1}{2} \frac{1}{c r}\left(c^{2} \tau_{r}(r)+E_{z c} r\left(\alpha_{c} c T_{c b}(r) \quad \alpha_{c} c T_{c t}(r)+2 w_{b}(r)\right.\right. \\
& \left.\left.2 w_{t}(r)+c^{2} D \tau_{r}(r)\right)\right)
\end{aligned}
$$

It should be noticed that for the case when the mechanical core properties are temperature dependent, the solution procedure fol lows the one that appears in Frostig and Thomsen [17], to which reference is made for brevity.

\section{Numerical study}

The numerical study presents the mechanical and the thermo mechanical non linear response of a circular sandwich plate with a diameter of $300 \mathrm{~mm}$; the geometry of the plate appears in Fig. 2.

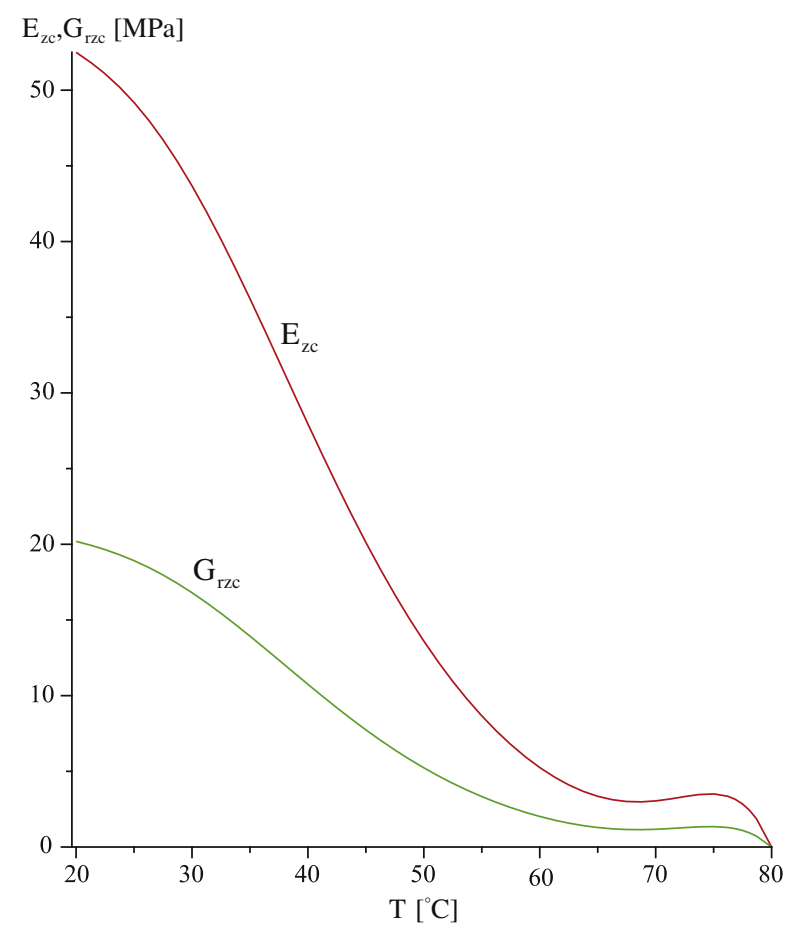

Fig. 3. Temperature dependence of elastic and shear moduli of PVC foam core, see Diab [2].
The sandwich plate is assumed to consist of two quasi isotropic glass/epoxy face sheets with a thickness of $0.5 \mathrm{~mm}$, and a cross linked PVC foam core with elastic and shear moduli of $52.5 \mathrm{MPa}$ and 21.0 MPa, respectively, at $20^{\circ} \mathrm{C}$, and a thickness of $19.05 \mathrm{~mm}$. The glass/epoxy equivalent modulus of elasticity is assumed to be $27.4 \mathrm{GPa}$, and its coefficient of thermal expansion is $0.6 \mathrm{E} 5 \mathrm{C}$. The modulus of elasticity and the radial shear modulus of the PVC core are temperature dependent, and the variation of $E_{z c}(T), G_{c}(T)$ with temperature $(T)$ appear in Fig. 3 for a temperature range of $2080^{\circ} \mathrm{C}$, based on the data in Diab data sheets [2]. The PVC foam core coefficient of thermal expansion is taken as 35E $5 \mathrm{C}^{1}$ and is assumed to be constant.

The circular sandwich plate is assumed to be simply supported at the edge of the lower face sheet only, and is subjected to a mechanical load that consists of a partially distributed load applied to a centric circle area of $40 \mathrm{~mm}$ diameter, see Fig. 3. The thermal load comprises of a linear thermal gradient of $\Delta T=040^{\circ} \mathrm{C}$ through the depth of the core and constant through the depth of each face sheet, see temperature distribution in Fig. 2. In addition the temperature field is assumed to be uniformly distributed throughout the plate in the face sheets and the core. The tempera ture at the upper face sheet is increased from $20^{\circ} \mathrm{C}$ until the lower face sheet reaches a temperature of $79{ }^{\circ} \mathrm{C}$.

\section{Finite element analysis (FEA) model}

Two FEA models have been developed using the ABAQUS/Stan dard commercial FEA code; a two dimensional (2D) model using axi symmetric elements, and a three dimensional (3D) model using eight node solid elements (C3D8R in Abaqus). The models include geometric non linearity and large deformations options, ABAQUS/Standard uses the Newton method to solve the non linear equilibrium equations using an incremental procedure with limit ing incremental size. The solution is obtained as a series of incre ments, with iterations to obtain equilibrium within each increment. Increments must be kept small to ensure correct mod eling of history dependent effects. Newton method has a finite ra dius of convergence; too large an increment can prevent any solution from being obtained because the initial state is too far away from the equilibrium state that is being sought, it is outside the radius of convergence. Thus, there is an algorithmic restriction on the increment size.

Moreover, the elastic and shear moduli of the core are defined as temperature dependent. Thus, the mechanical properties are de graded according to the temperature field imposed at each node. In the 3D model only a quarter of the circular sandwich plate was modeled due to the symmetry of the problem, see Fig. 4 . The face sheets and the core are fully bonded, and to avoid that the core and 
(a)

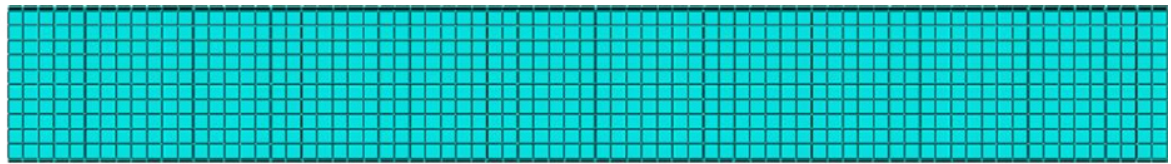

(b)

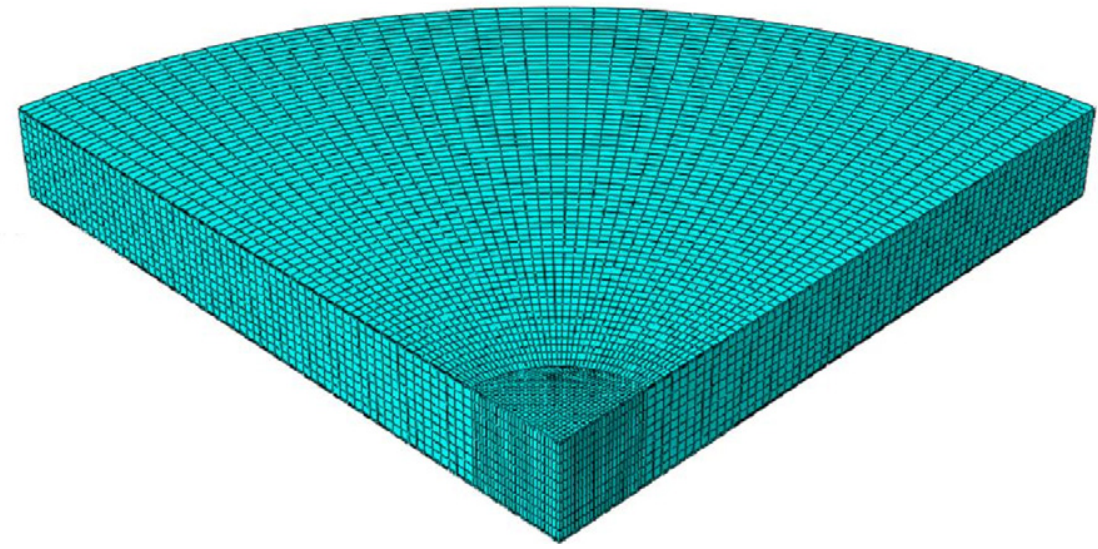

Fig. 4. Sandwich circular plates FEA models: (a) final mesh for the $2 \mathrm{D}$ axi-symmetrical FEA model; (b) final mesh for the 3D FEA model.

face elements cross over each other during deformation, the nodes of the interface are shared by the elements of the face sheets and the core. Hence, when large stiffness differences exist between the shared elements numerical instabilities and convergence diffi culties occur. Non uniform increment sizes are considered since the stiffness ratio between the core and face sheet materials is changed for each increment. The increment sizes are chosen to maintain small rotations and strain increments. The increment size decreases as the temperature is increased to avoid (or minimize) the occurrence of numerical instabilities and lack of convergence due to the degradation of foam core properties. The choice of incre ment size affects the computation efficiency. Hence, too large increments require more iterations, which on the other hand needs a refinement of the mesh in the vicinity of the distorted element to reduce the total computation time. According to this procedure, successive space discretizations have been carried out to evaluate the sensitivity of the mesh. Finally, the selected meshes has 1050 four node axi symmetric elements with reduced integration (CAX4R in Abaqus) in the axi symmetric model, and 48,664 eight node brick elements with reduced integration (C3D8R in ABAQUS) in the 3D model, final meshes appear in Fig. 4. In both models, the axi symmetric and the 3D models, four elements are used through the thickness of the face sheets, and ten elements are used through the core thickness. A uniform mesh is used in the axi symmetric model due to the low number of elements; however a refined mesh in the vicinity of the loading circle and the edges is necessary for the 3D model due to the accumulation of large stresses in the vicinity of the supports. It should be noticed that the sandwich plate is supported only along the rim of the low er face sheet.

The HSAPT model is based on the assumption that the through thickness the vertical modulus of elasticity and the shear modulus are non zero, whereas the in plane stiffness can be ignored. Effec tively, this requires vertically orthotropic core properties. This hypothesis is adopted in the HSAPT formulation to allow a closed form solution for the core displacement and stress fields, and it is physically plausible since the in plane stiffness of the face sheets is much higher than that of the core and the couple formed in the section of the panel is almost totally being carried by the face sheets. In the FEA model it is assumed that in plane elasticity and shear moduli are non zero and correspond to the mechanical properties that appear in the data sheet of Diab [2].
In order to verify this assumption of the HSAPT model the FEA models include both cores with isotropic and orthotropic mechan ical properties. In the case of an orthotropic core the in plane stiff ness is reduced to correlate with the HSAPT hypothesizes. The differences in predictions when assuming either isotropic or ortho tropic foam core properties are evaluated to estimate the influence of the in plane core stiffness on the behavior of the sandwich plate.

The numerical modeling of foam cored sandwich structures re quires a thorough convergence study in order to achieve a reliable solution. Hence, it is necessary the use of an implicit finite element method which allows to limit the error in each increment. The mesh refinement procedure must be developed along with the determination of the increment size. Once a sufficient refined mesh is defined the increment size must be reduced until the solution reaches a stable value. The model must be subjected to numerical iterations in order to achieve stability of the solution when a reduction in the increment size or a refinement of the mesh is used. Notice that the convergence study has to be repeated for each temperature. Since the stiffness ratio between the face sheets and core materials increase as the temperature are raised as a result of the degradation of the core properties. This procedure leads to an inevitable balance between the precision in the solution and the computational resources required. Thus the comparison with other solutions, analytical or experimental results is very useful in order to reach an accurate solution involving low computational re sources. If alternative solutions are not available, the convergence study must be carried out carefully to ensure that the FEA simula tions converge to a stable solution. To achieve a converged solution for the case of foam cored sandwich structures subjected to ther mo mechanical loading, it is a prerequisite that the researcher pos sesses high expertise and skills in FEA modeling, and also possesses deep understanding of the mechanics of sandwich structures, espe cially when a low strength core that degrades with the rising tem perature is used.

\section{Numerical results}

In the forthcoming, the FEA model results are based on the assumption that the orthotropic core properties consists of an in plane elasticity and shear moduli that are $20 \%$ of those of the ver tical one (i.e. $E_{r}=E_{\theta}=E_{z c} / 5$ and similar with the shear modulus). 
The results obtained with the axi symmetric (2D) and the 3D FEA models are compared, and they are almost identical, see Fig. 5.

The deformed shapes of a circular sandwich plate loaded by a partially distributed load ( $d=40 \mathrm{~mm}$, see Fig. 2$)$ at various load levels of the HSAPT model appear in Fig. 6. It is observed that the displacements patterns for the different load levels are very similar and they increase as the load increases. The deformed shapes ob tained from the FEA models, and the HSAPT models are similar, see Figs. 5 and 6.
The load vs. the maximum vertical displacements at the plate centre appears in Fig. 7a. The results include the vertical displace ments at the upper and lower face sheets of the axi symmetric FEA model. The FEA model exhibits stiffer behavior compared with the HSAPT model, and thus the FEA models yields smaller deflection predictions. The reason for this is that a discrete finite element model is always stiffer than the real member, moreover while in the HSAPT model the in plane stiffness is neglected, the in plane stiffness in the FEA model is reduced but not stated as zero.

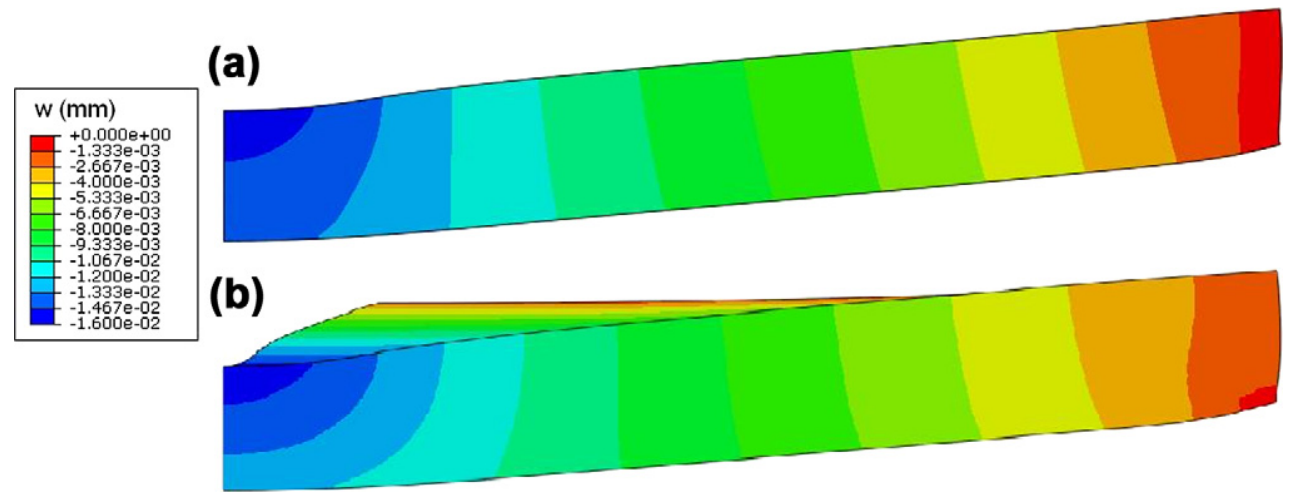

Fig. 5. FEA vertical displacement fields due to a distributed load of $2 \mathrm{MPa}$ and temperature filed of at $T_{b}=79{ }^{\circ} \mathrm{C}$ and $T_{t}=59{ }^{\circ} \mathrm{C}$ with a linear gradient: (a) axi-symmetric (2D) FEA model. (b) 3D FEA model.

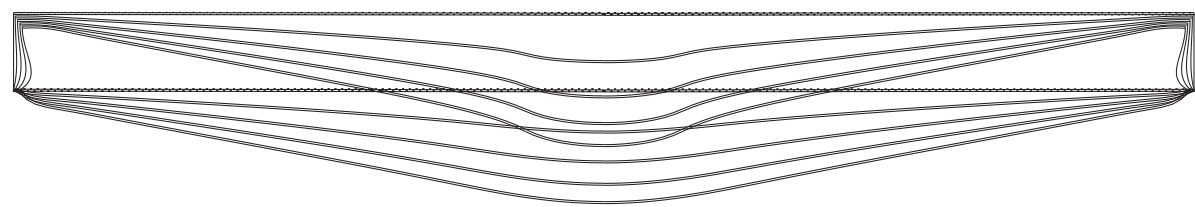

Fig. 6. HSAPT predictions: deformed shapes of a sandwich panel loaded by a partially distributed load $(d=40 \mathrm{~mm})$ at various load levels.
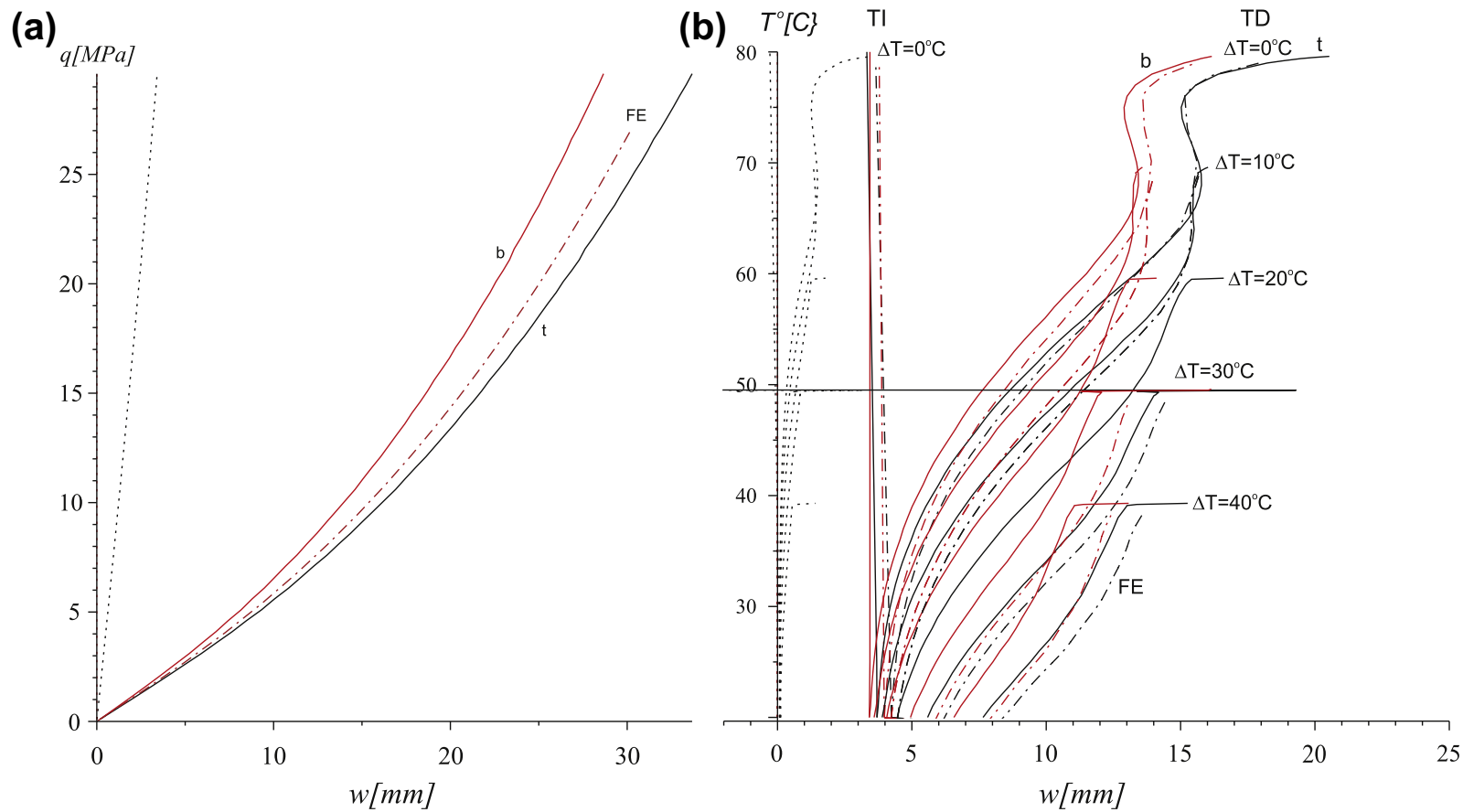

Fig. 7. Load and temperatures vs. extremum vertical displacements of the face sheets (HSAPT and FEA results): (a) fully distributed load only; (b) partially distributed load $(d=40 \mathrm{~mm})$ and thermal loading of a sandwich plate with TI and TD properties). Legend: upper face sheet (HSAPT) lower face sheet (HSAPT), _._ FEA. 


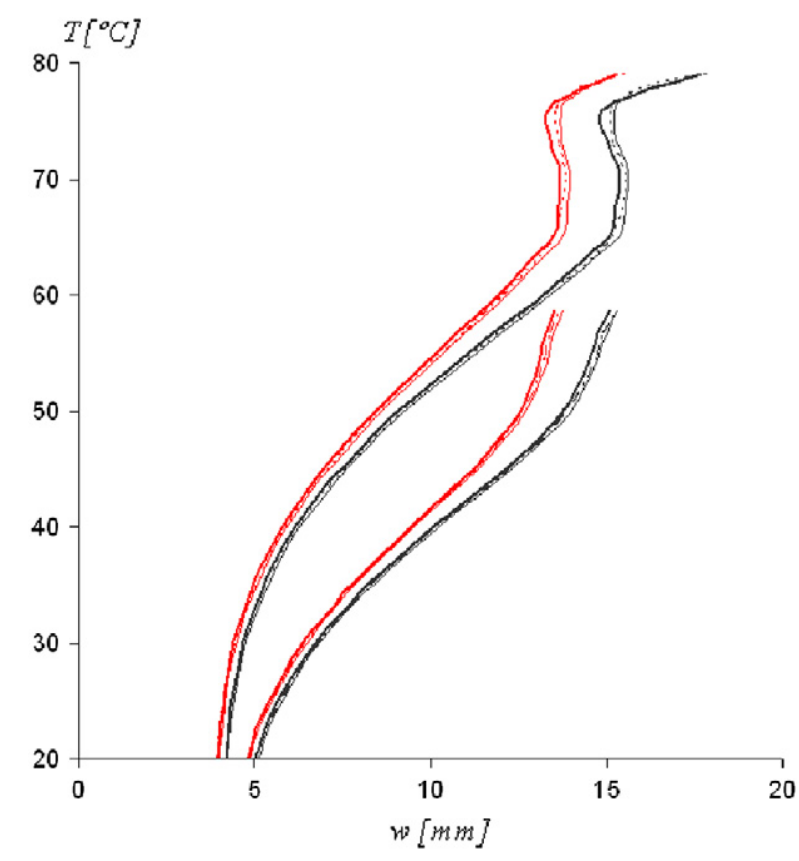

Fig. 8. FEA results of temperatures vs. extreme vertical displacements of face sheets (FEA results) for a partially distributed load $(d=40 \mathrm{~mm})$ and thermal loading with TD properties. Legend: — isotropic core with $E_{r}=E_{\theta}=E_{z c}$, _ orthotropic core: $E_{r}=E_{\theta}=E_{z c} / 5, \ldots$ orthotropic core: $E_{r}=E_{\theta}=E_{z c} / 10$.

It should be noticed, however, that the predicted displacements using the HSAPT and FEA models are very similar. It should further be noticed, that the low out of plane stiffness of the core yields an indentation in the vicinity of the mid span loading area leading to different displacements of the upper and lower face sheets.

The equilibrium curves corresponding to a circular sandwich plate subjected to a distributed load of $2.0 \mathrm{MPa}$ appear for all temperature gradients with temperature dependent (TD) core properties in Fig. 7b, which also includes the case of a uniform temperature distribution with the assumption of temperature independent $(\mathrm{TI})$ core properties. The equilibrium curves based on the HSAPT approach have been determined using a parametric continuation procedure up to the temperature level when either the solution does not converge, or the upper limit of the range of the operating temperatures $\left(79^{\circ} \mathrm{C}\right)$ has been reached. Hence, there is no case where the core stiffness reaches the value of zero within the core depth. The temperature of the upper face sheet is plotted as a function of the maximum vertical displacements at the upper and the lower face sheets, assuming a core attributed with either TD or TI properties.

The results obtained from the FE model are in close agreement with the results of the HSAPT analysis. Fig. 7b reveals that the TI core with a zero thermal gradient yields a linear curve which indi cates a stable behavior within this range of temperatures. In con trast, the TD cases behavior yield an unstable non linear response, and in all cases the upper limit of the temperature range has been reached.

The case with null thermal gradient $\left(\Delta T=0^{\circ} \mathrm{C}\right)$ is the most sen sitive one; it consists of a stable curve up to about $78^{\circ} \mathrm{C}$, and above it loss of stability occurs. The HSAPT results reveal also that when the maximum temperature is reached at the lower face sheet, the circular plate becomes unstable as a result of the degradation of the stiffness of the core. However, the FE analysis procedure ceased to converge and thus halted just before the maximum temperature is reached due to numerical instabilities caused by the large distortions in the elements of the core. The reason for the poor "numerical performance" of the FEA solver is that the ratio between the E moduli of the face sheets and the core is above 300 at room temperature, and this ratio becomes significantly large as the temperature increase. This difference between the stiffness of adjacent elements led to poor convergence and severe numerical difficulties. In overall the FEA and the HSAPT results correlate very well.

In order to quantify the effects of the in plane stiffness of the core on the plate response three FEA models are presented: isotro pic and orthotropic with various in plane to vertical modulus ratios, $1 / 5$ and $1 / 10$ that correspond to the HSAPT model, see Fig. 8. It reveals that the equilibrium curves of the isotropic foam core are in excellent correlation with the results of the plates with the orthotropic cores. Accordingly, Fig. 8 shows that the influence of the core in plane stiffness on the response of foam cored sandwich circular plates under thermo mechanical loads can be neglected as assumed by the HSAPT approach for this particular construction.

\section{Summary and conclusions}

The non linear behavior of a circular sandwich plate with a compliant core and temperature dependent core properties has been investigated through analytical, HSAPT computational model and FEA analyses. The analyses include the induced thermal dis placements along with the effects of the vertical flexibility of the core. The HSAPT governing equations in the case of a core with temperature independent properties are presented. The tempera ture dependent properties of the core, along with the presence of a through thickness thermal gradient, yields core stiffness proper ties that vary through the core thickness and they have been solved analytically the HSAPT approach. Two FEA models have been developed using the ABAQUS/Standard code, a 2D axisymmetrical model, and a 3D model to analyze the geometrical non linear behavior of a sandwich plate with a compliant core and tempera ture dependent core properties.

The use of FEA codes to model the behavior of polymer foam cored sandwich structures subjected to mechanical loading and elevated temperatures requires advanced techniques due to numerical instabilities and lack of convergence that are caused by the degradation of the core mechanical properties. These diffi culties are a result of the distortion of the elements at the interface between face sheets and core due to the large difference in their stiffnesses. These difficulties increase as the core stiffness degrades (decrease) as a result of increasing temperature.

Based on the work conducted and presented herein, a few gen eral recommendations can be given regarding the FEA modeling of foam cored sandwich structures subjected to thermo mechanical loading:

- Great care should be shown with respect to chosen element types and mesh density adopted for both the faces and the core material. The thin faces would typically require the use of sev eral elements through the thickness to capture the characteris tic short wave length local bending responses that appears near supports and load introduction points.

- The use of an implicit finite element method is recommended, as this allows to limit the error in each solution increment.

- Large element deformations should be considered.

- Compatible finite elements should be chosen for the faces and the core to avoid problems with "over crossing" element bound aries, or penetration of the face sheet elements into the soft core, at the face core interfaces.

- A complete convergence study is required in each temperature step to ensure that a sufficiently refined FE mesh is achieved and a reliable solution has been determined. 
- Different increment sizes must be used for each temperature because the stiffness ratio between face sheets and core mate rials depends on the degradation (reduction) of the mechanical core properties with increasing temperature.

The numerical study of the non linear response consists of two parts. In the first part, an axi symmetric partially distributed mechanical load is applied to a circular sandwich plate that is sim ply supported at its lower face sheet while the upper face and the core are free. The second part examines the effect of the rising fully distributed temperatures along with the partially distributed load on the response.

The response due to mechanical loading only of the HSAPT and FEA models in terms of load vs. mid span displacement are similar. However, the FEA model exhibits a somewhat stiffer behavior than the HSAPT model. Both the HSAPT and FEA models show that the low stiffness of the foam core forms an indentation in the vicinity of the partially distributed mechanical load that is associated with significant differences between the displacements of the upper and lower face sheets.

The interaction between the mechanical loading and rising tem perature has been studied using the HSAPT and FEA approaches with TI and TD core properties. The HSAPT model predictions for circular sandwich plates compared well with the FEA analysis re sults expressed in terms of temperature vs. mid span displace ments for different through core thickness thermal gradients $\left(\Delta T=040^{\circ} \mathrm{C}\right)$. However, the FEA analyses are associated with se vere numerical difficulties and poor convergence as a result of the large distortions of the finite elements of the core caused by the degradation (reduction) of the core mechanical properties with ris ing temperature.

The comparative study (HSAPT vs. FEA) of the interaction be tween mechanical loading and rising temperature results demon strates that the response for an unrestrained circular sandwich plate with a TI core remains linear and constant through the entire range of working temperatures. In contrast, the response in the case of a TD core, which causes degradation of the properties, is strongly non linear for all through thickness thermal gradients. The case with a uniform temperature distribution is the most se vere case and it is associated with loss of stability when the tem perature reaches near the temperature when loss of strength occurs, at about $79^{\circ} \mathrm{C}$.

The validity of the HSAPT assumption of neglected in plane stiffness of the foam core is confirmed by the comparison of the FE model results considering isotropic and orthotropic, with vari ous in plane to vertical modulus of elasticity ratios, foam core properties. The differences in the results between the plates with isotropic core properties or orthotropic ones are negligible. Hence, the influence of the foam core in plane stiffness can be neglected.

The interaction between mechanical and a thermally induced deformation loads is highly relevant and likely to occur in foam cored sandwich plates subjected to practical service conditions. This is especially the case for sandwich plates with temperature dependent core properties (as is always the case for polymer foam core materials), and such interactions may seriously affect the structural reliability and safety. Hence, it is important to assess these effects for a reliable design of sandwich plate structures. This implies that a non linear analysis procedure, which accounts for the compliant temperature dependent core properties and the interaction between the mechanical loads and thermally induced deformations must be adopted.

\section{Acknowledgments}

This work presented in this paper was conducted during the stays of Dr. Carlos Santiuste and the visit of Professor Yeoshua Fro stig with the Department of Mechanical and Manufacturing Engi neering at Aalborg University.

The stay of Dr. Santiuste was sponsored by the Department of Continuum Mechanics and Structural Analysis, University Carlos III of Madrid, Spain. The visiting professorship of Professor Frostig was sponsored by the US Navy, Office of Naval Research (ONR), Award N000140710227, "Influence of local effects in sandwich structures under general loading conditions and ballistic impact on advanced composite and sandwich structures", where the pro grammer manager was Dr. Yapa D.S. Rajapakse and by the Ashtrom Engineering Company, which supports Frostig's professorial chair at Technion Israel Institute of Technology. The financial support received is gratefully acknowledged.

\section{References}

[1] Zenkert D. The handbook of sandwich construction. UK: EMAS Publishing; 1997.

[2] Divinycell. Data Sheet Hd Grade 2003; Diab Group <www.diabgroup.com>.

[3] Evonik Röhm Gmbh. WF Foam Data sheets 2004; <www.rohacell.com>.

[4] Mindlin RD. Influence of transverse shear deformation on the bending of classical plates. J Appl Mech 1951;18:31-8.

[5] Reddy JN. Energy and variational methods in applied mechanics. New York: Wiley; 1984.

[6] Najafizadeh MM, Heydari HR. Thermal buckling of functionally graded circular plates based on higher-order shear deformation plate theory. Eur J Mech A/ Solids 2004;23:1085-100.

[7] Dafedar JB, Desai YM. Thermomechanical buckling of laminated composite plates using mixed, higher-order analytical formulation. J Appl Mech, Trans ASME 2002;69:790-9.

[8] Kapuria S, Achary GGS. An efficient higher-order zigzag theory for laminated plates subjected to thermal loading. Int J Solids Struct 2004;41:4661-84.

[9] Shiau LC, Kuo SY. Thermal postbuckling behavior of composite sandwich plates. J Eng Mech, ASCE 2004;130(10):1160-7.

[10] Matsunaga H. Thermal buckling of cross-ply laminated composite and sandwich plates according to a global high-order deformation theory. Compos Struct 2005;68:439-54.

[11] Dawe DJ. Use of finite strip method in predicting the behavior of composite laminated structures. Compos Struct 2002;57:11-36.

[12] Cooke GME. Stability of lightweight structural sandwich panels exposed to fire. Fire Mater 2004;28:299-308.

[13] Frostig Y. Hygrothermal (environmental) effects in high-order bending of sandwich beams with a flexible core and a discontinuous skin. Compos Struct 1997;37(2):205-21.

[14] Frostig Y, Thomsen OT. Localized effects in the non-linear behavior of sandwich panels with a transversely flexible core. J Sandwich Struct Mater 2005;7(1):53-75.

[15] Frostig Y, Thomsen OT, Sheinman I. On the non-linear high-order theory of unidirectional sandwich panels with a transversely flexible core. Int J Solids Struct 2005;42(5-6):1443-63.

[16] Frostig Y, Thomsen OT. Thermal buckling and post-buckling of sandwich panels with a transversely flexible core. AIAA J 2008;46(8):1976-89.

[17] Frostig Y, Thomsen OT. Buckling and non-linear response of sandwich panels with a compliant core and temperature-dependent mechanical properties. J Mech Mater Struct 2007;2(7):1355-80.

[18] Frostig Y, Thomsen OT. Non-linear thermal response of sandwich panels with a flexible core and temperature dependent mechanical properties. Composites Part B 2008;39(1):165-84.

[19] Frostig Y, Thomsen OT. Non-linear geometrically thermal response of circular sandwich plates with a compliant core, submitted for publication. 